\title{
Reproducibility of optic disk evaluation in supine subjects with a Heidelberg Retina Tomograph II laser tomographic scanner
}

This article was published in the following Dove Press journal:

Clinical Ophthalmology

24 August 2016

Number of times this article has been viewed

\author{
Yosuke Harada' \\ Tomoyuki Akita ${ }^{2}$ \\ Joji Takenaka' \\ Yuko Nakamura-Kadohiro' \\ Junko Tanaka ${ }^{2}$ \\ Yoshiaki Kiuchi' \\ 'Department of Ophthalmology and \\ Visual Science, Graduate School of \\ Biomedical Sciences, ${ }^{2}$ Department \\ of Epidemiology, Infectious Disease \\ Control and Prevention, Institute \\ of Biomedical and Health Sciences, \\ Hiroshima University, Hiroshima, Japan
}

Correspondence: Yosuke Harada Department of Ophthalmology and Visual Science, Graduate School of Biomedical Sciences, Hiroshima University, I-2-3 Kasumi, Minami-Ku, Hiroshima 734-855I, Japan

Tel +81822575247

Fax +8I 822575249

Email yyaounde@gmail.com
Aim: The aim of this study was to determine the reproducibility and reliability of optic disk evaluation in supine subjects with a Heidelberg Retina Tomograph II (HRT II).

Methods: One eye was randomly selected for evaluation by HRT II in each of eleven healthy subjects. Five images were obtained by each of two experienced operators at two separate visits with the subjects in both sitting and supine positions. A stand was constructed to allow stable, handheld operation of the HRT II head for imaging of the supine subjects. Measurements of optic disk parameters obtained in the supine position were compared with those obtained in the sitting position. The reliability of measurements in the supine position was evaluated by calculating both the coefficients of variation for measurements made by one observer on the first visit and the interobserver and intervisit intraclass correlation coefficients (ICCs); the values obtained were compared with those obtained for the sitting position.

Results: Measurements of all parameters made in the supine position were highly similar to those made in the sitting position. The coefficients of variation obtained for each parameter in the sitting and supine positions ranged from $5.67 \%$ to $14.16 \%$ and from $2.18 \%$ to $16.08 \%$, respectively. The interobserver ICC values in the sitting and supine positions were $\geq 0.978$ and $\geq 0.989$, respectively. The intervisit ICC values in the sitting and supine positions were $\geq 0.958$ and $\geq 0.983$, respectively.

Conclusion: Handheld operation of the HRT II in a custom-designed stand is feasible for optic disk evaluation in the supine subjects who were not able to maintain the sitting position.

Keywords: glaucoma, optic disk evaluation, supine position, Heidelberg retina tomograph

\section{Introduction}

Assessment of optic disk plays a key role in the diagnosis and care of individuals with glaucoma. The changes in optic disk structure may precede the onset of a measurable visual field defect, ${ }^{1-3}$ hence evaluation of the optic disk is important for the early detection of glaucoma. Recording of the optic disk image, in addition to visual field tests, is required for effective monitoring of glaucoma patients over a period of time. Objective analysis of the optic disk is of particular value for the assessment of patients who cannot be readily tested for visual field, such as young children with developmental glaucoma.

The Heidelberg Retina Tomograph II (HRT II) has been used to three-dimensional evaluation of the optic disk. ${ }^{4,5}$ In its present form, however, this instrument cannot be used for subjects in the supine position. Measurement of the optic disk in the supine position with the HRT II is theoretically possible, since the laser unit can be detached from the stand and used as a handheld camera. If it could be shown to be reproducible 
and reliable, this approach would provide objective information on the optic disk in individuals who cannot maintain a sitting position.

We have evaluated the reproducibility and reliability of the measurement of optic disk parameters with the HRT II in individuals in the supine position. We have also evaluated whether these measurements correlate with those obtained in the sitting position.

\section{Methods}

\section{Participants}

The study was approved by the institutional review board of Hiroshima University and conformed to the tenets of the Declaration of Helsinki. Eleven healthy subjects were recruited by poster advertisement at Hiroshima University. Written informed consent was obtained from all the participants. All subjects underwent ophthalmic assessment, including measurement of visual acuity and intraocular pressure, anterior segment examination by slit-lamp biomicroscopy, and fundus examination to confirm the absence of manifest ophthalmic disease. One eye was selected at random for each subject; a refractive error of $<10 \mathrm{D}$ of spherical power was required for inclusion in this study, as clear HRT II images cannot be obtained for eyes with severe myopia.

\section{Optic disk evaluation by HRT II with subjects in the supine position}

For evaluation of subjects in the supine position, the laser unit of the HRT II was used as a handheld camera. A fixing device was therefore needed both to avoid the blurring of images caused by shaking of the operator's hand and/or movement of the subject's head and to maintain an appropriate distance $(10 \mathrm{~mm})$ between the subject's cornea and the laser unit. This device was constructed from an acrylic board and two pieces of polystyrene foam (Figure 1). The board had two holes: one with a diameter of $3 \mathrm{~cm}$ that was aligned with the apex of the laser unit held between the two pieces of polystyrene and the other coincident with the subject's nose to prevent it from pressing up against the board. Each of the two polystyrene blocks had a recess of $6.5 \times 6.5 \times 9.5 \mathrm{~cm}$ and was attached to the acrylic board in order to fix the laser unit in place. The board was placed on an extendable surgical wrist rest (Handaya, Tokyo, Japan) to maintain the appropriate distance between the subject's cornea and the laser unit; the laser unit was positioned in the recess of the polystyrene blocks to prevent image blurring due to hand movement.

\section{Testing protocol and statistical analysis}

One eye was selected randomly for each subject. All HRT II images were obtained by each of two experienced operators (observers 1 and 2) both on a first visit and on a second visit within 2 weeks of the first visit. The HRT images were acquired without pupil dilation. Each operator acquired an HRT II image five times at each visit with the subject first in the sitting position and then in the supine position. After collection of the images with the subject in the sitting position, the subject was instructed to lie on a bed for 5 minutes, and the images were then obtained in the supine position.
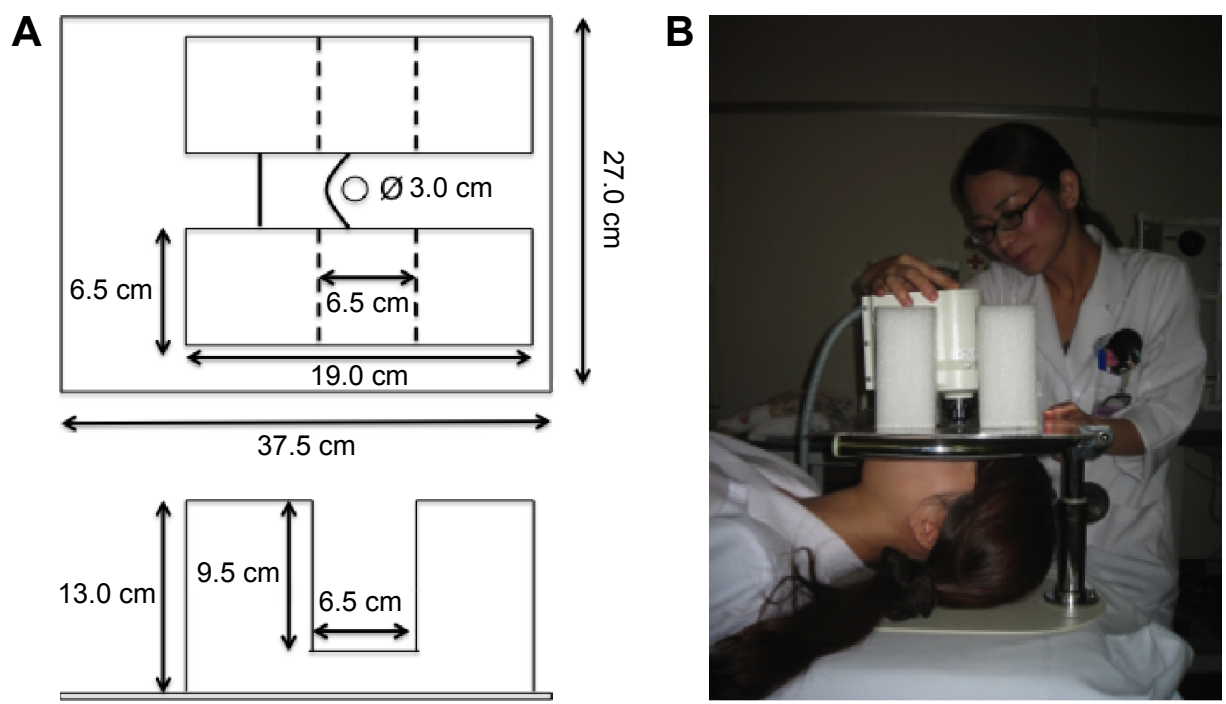

Figure I Measurement of optic disk parameters with the subjects in the supine position by HRT II.

Notes: (A) An acrylic board with two attached pieces of polystyrene foam was constructed to hold the laser unit of the HRT II. Each polystyrene block had dimensions of $6.5 \times 19.0 \times 13.0 \mathrm{~cm}$ and had a recess of $6.5 \times 6.5 \times 9.5 \mathrm{~cm}$ to hold the laser unit. Between the two pieces of polystyrene, the acrylic board had two holes, one with a diameter of $3 \mathrm{~cm}$ aligned with the apex of the laser unit and the other aligned with the subject's nose. (B) The acrylic board was placed on a surgical wrist rest, and the laser unit was positioned in the recess of the polystyrene blocks, to allow collection of HRT II images with the subject in the supine position.

Abbreviation: HRT II, Heidelberg Retina Tomograph II. 
The disk margin was defined, by observer 1 , as a contour line in the first stored tomographic image obtained in the sitting position. This contour line was transposed automatically to the remaining images obtained in both the sitting and supine positions. Six stereometric parameters - cup area (CA), rim area (RA), cup/disk area (C/D) ratio, cup volume (CV), rim volume (RV), and mean cup depth (MCD) - were compared between the sitting and supine positions.

To test for correlation between parameters measured in the supine position and those measured in the sitting position, we compared the average and the coefficient of variation ( $\mathrm{SD} / \mathrm{mean}$ ) for each parameter obtained by observer 1 on the first visit. The agreement between measurements obtained in the sitting and supine positions was further graphically evaluated with the use of Bland-Altman analysis. In this analysis, the distribution of measurements was expressed as the mean difference between the supine and sitting positions and in terms of the $95 \%$ limits of agreement, which were defined as the mean \pm 1.96 SD. ${ }^{6,7}$ Fixed bias and proportional bias were estimated. Fixed bias was determined to evaluate whether the measurements in the supine position are higher or lower than those in the sitting position by a constant amount. Proportional bias was determined to evaluate the effect of disk parameters on the agreement between the values obtained in the two positions. The presence of proportional bias was evaluated by regression analysis of the difference between the values obtained in the sitting and supine positions. These analyses were performed with the data obtained by observer 1 on the first visit.

To estimate the reproducibility and reliability of measurements obtained in the sitting and supine positions, we also calculated the intraclass correlation coefficient (ICC) for interobserver (different observer/same day) and intervisit (same observer/different day) conditions. The acquisition protocol was as described previously, ${ }^{8}$ and the analysis was performed with IBM SPSS Statistics for Windows, Version 19.0, software (IBM Corporation, Armonk, NY, USA). All other statistical analyses were performed with JMP, Version 7.0, software (SAS Institute Inc., Cary, NC, USA), and $P$-values of $<0.05$ were considered statistically significant.

\section{Results}

Six men and five women with a mean \pm SD age of $31.1 \pm 4.2$ years (range, 26-37 years) were included in this study. All participants were healthy individuals, without glaucoma. Slit-lamp examination showed that all participants' disks were healthy, without any abnormality, such as peripapillary atrophy. The averages for each subject's parameters (CA, $\mathrm{RA}, \mathrm{C} / \mathrm{D}$ ratio, $\mathrm{CV}, \mathrm{RV}$, and $\mathrm{MCD}$ ) measured five times in the supine position by observer 1 on the first visit were compared with those obtained in the sitting position (Figure 2). All parameters measured in the supine position were highly
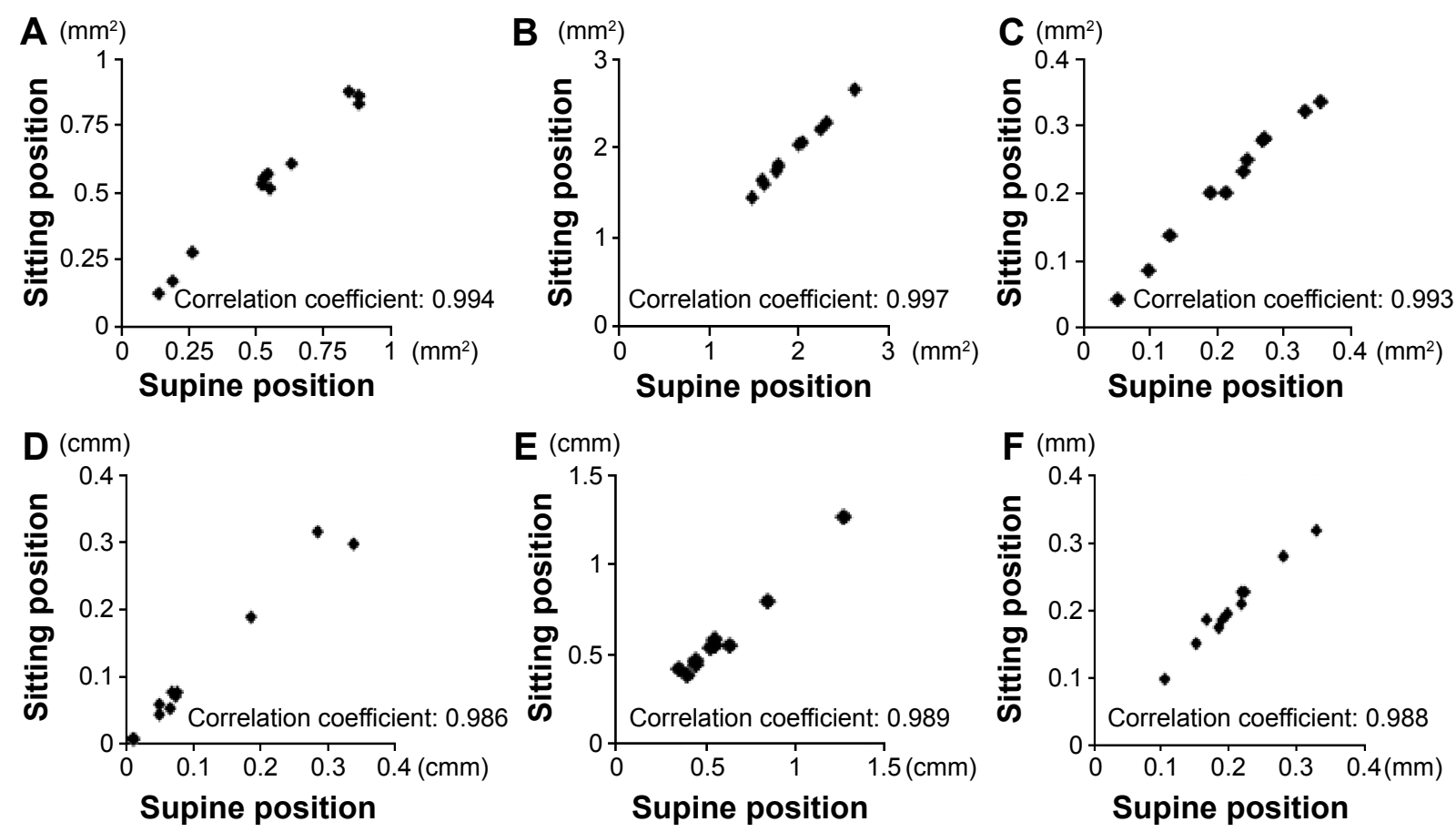

Figure 2 Correlation between the average disk parameter values obtained from the subjects in the sitting and supine positions.

Notes: The averages of the five measurements of (A) CA, (B) RA, (C) C/D ratio, (D) CV, (E) RV, and (F) MCD made by observer I for each subject on the first visit were compared between the supine and sitting positions. The correlation coefficient $(r)$ is shown for each comparison.

Abbreviations: CA, cup area; RA, rim area; C/D, cup/disk area; CV, cup volume; RV, rim volume; MCD, mean cup depth. 
similar to those measured in the sitting position, with the correlation coefficients ranging from 0.986 to 0.997 .

Bland-Altman plots were applied to investigate the existence of any systematic difference between measurements obtained in the sitting and supine positions (Figure 3). The average for each parameter measured in the two conditions was plotted on the $x$-axis, and the difference between the two measurements (supine and sitting) was plotted on the $y$-axis. The mean difference was small for all parameters, and all limits of agreement included the value 0 , indicating the absence of fixed bias. The measurements obtained in the sitting and supine positions also did not show proportional bias, as indicated
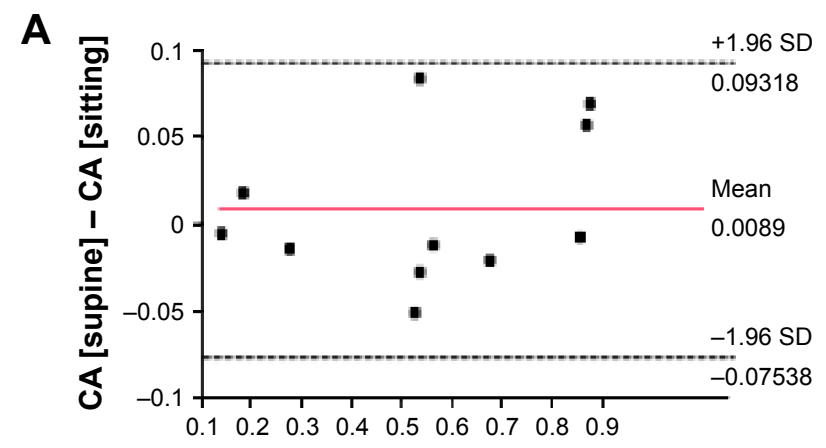

(CA [supine] + CA [sitting])/2

C

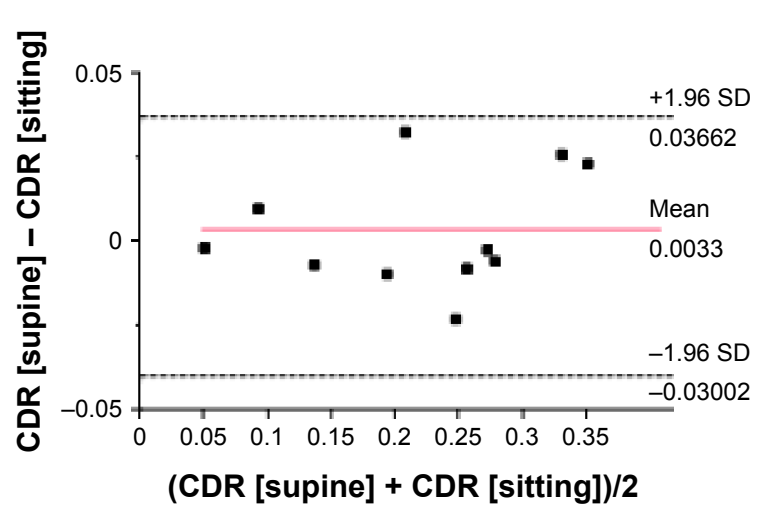

E

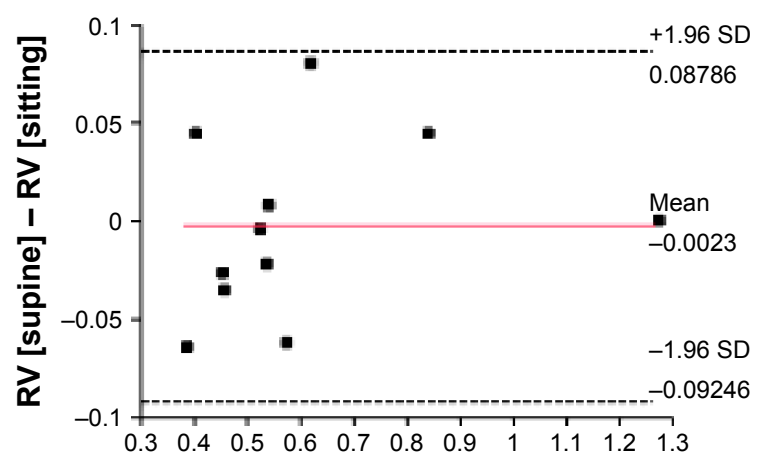

(RV [supine] + RV [sitting])/2 by a nonsignificant slope of the regression line ( $P$-values of 0.3599, 0.8250, 0.4769, 0.5639, 0.4568, and 0.9982 for CA, $\mathrm{RA}, \mathrm{C} / \mathrm{D}$ ratio, $\mathrm{CV}, \mathrm{RV}$, and $\mathrm{MCD}$, respectively).

The coefficients of variation for the five measurements of each parameter made by observer 1 on the first visit with the subjects in the sitting and supine positions ranged from $5.67 \%$ to $14.16 \%$ and from $2.18 \%$ to $16.08 \%$, respectively (Table 1). The highest observed value was for CV in each position, whereas the lowest was for RA in the supine position and for MCD in the sitting position.

Finally, determination of the ICC for interobserver and intervisit conditions revealed that the measurement of all

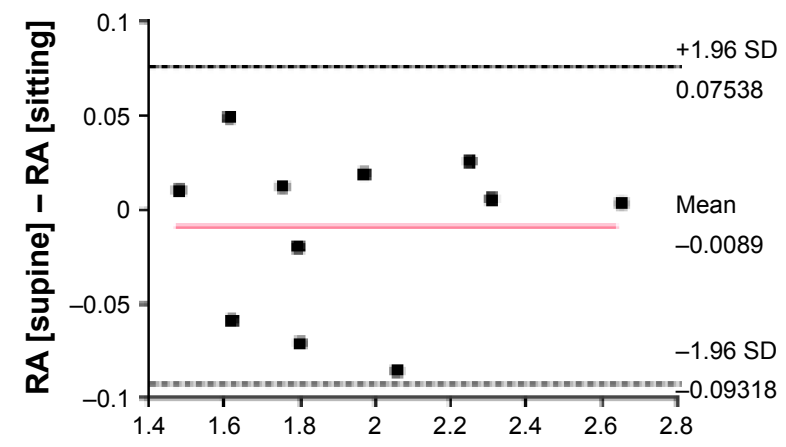

(RA [supine] + RA [sitting])/2
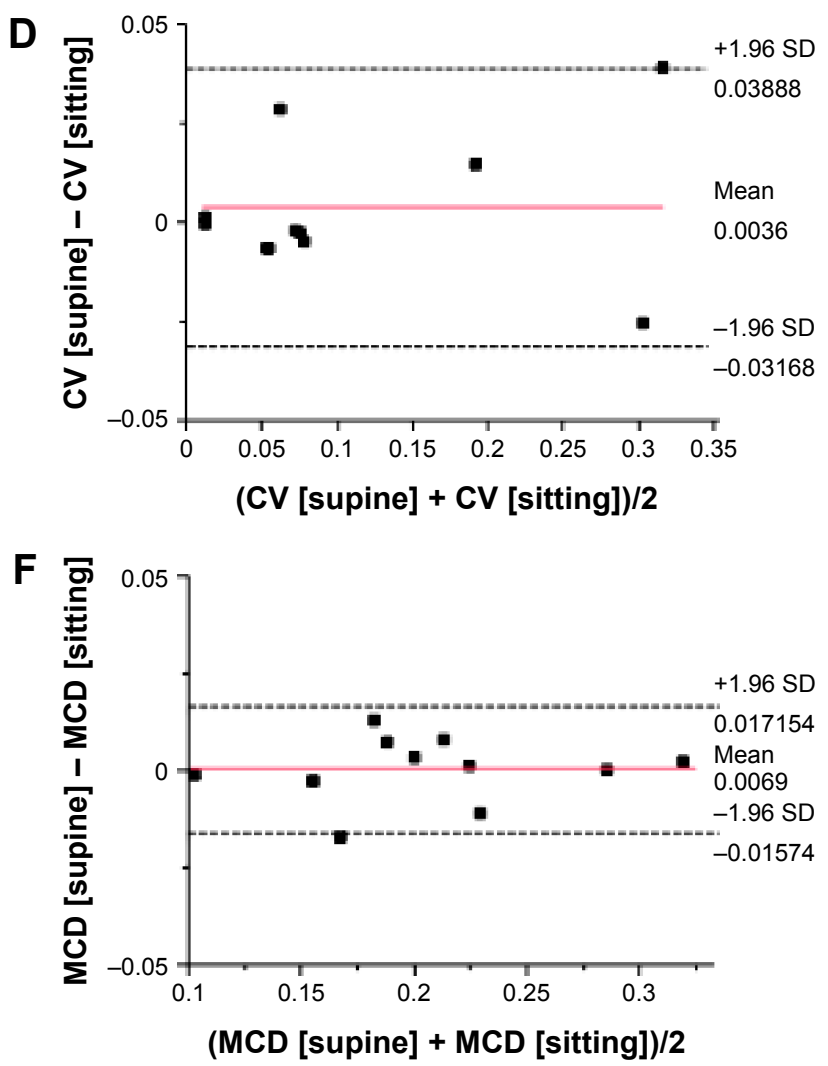

Figure 3 Bland-Altman plots for disk parameters of each subject measured in the supine position versus sitting position.

Notes: The plots were generated from the averages of the five measurements of (A) CA, (B) RA, (C) CDR, (D) CV, (E) RV, and (F) MCD made by observer I for each subject in the supine and sitting positions on the first visit. The mean and mean $\pm 1.96 \mathrm{SD}$ values on the $y$-axis are indicated by red and dashed lines, respectively. Abbreviations: CA, cup area; RA, rim area; C/D, cup/disk area; CV, cup volume; RV, rim volume; MCD, mean cup depth; CDR, C/D ratio; SD, standard deviation. 
Table I Coefficients of variation (\%) for the five measurements of stereometric parameters obtained by observer $I$ at the first visit for all subjects in the sitting and supine positions

\begin{tabular}{lll}
\hline Parameter & Sitting & Supine \\
\hline CA & 7.99 & 8.06 \\
RA & 7.23 & 2.18 \\
C/D ratio & 8.00 & 8.04 \\
CV & 14.16 & 16.08 \\
RV & 8.00 & 6.55 \\
MCD & 5.67 & 7.54 \\
\hline
\end{tabular}

Abbreviations: $C A$, cup area; RA, rim area; $C / D$, cup/disk area; $C V$, cup volume; $R V$, rim volume; $M C D$, mean cup depth.

parameters in the supine position was as highly reliable as that in the sitting position (Table 2). The ICC values for all parameters in both positions were $\geq 0.958$. With the exception of $\mathrm{CV}$, the interobserver ICC values for parameters measured in the supine position were equal to or greater than those obtained in the sitting position. With the exception of MCD, the intervisit ICC values for parameters measured in the supine position were greater than those in the sitting position.

\section{Discussion}

We have demonstrated here the reproducibility and reliability of optic disk evaluation by HRT II with the subject in the supine position. The laser unit of the HRT II was stabilized for such evaluation with a specially designed stand for handheld operation; the resulting measurements of optic disk parameters obtained with subjects in the supine position were virtually identical to those obtained in the sitting position.

In this study, the coefficient of variation and ICC values for measurements obtained with the subjects in the supine position were highly similar to those obtained in the sitting position. Previous HRT studies have analyzed data for optic disk parameters measured only in the sitting position. One such study showed that, for the same six optic disk parameters

Table 2 Interobserver and intervisit ICC values for measurement of stereometric parameters with subjects in the sitting and supine positions

\begin{tabular}{llllll}
\hline Parameter & Sitting & & & \multicolumn{2}{l}{ Supine } \\
\cline { 2 - 3 } \cline { 5 - 6 } & Interobserver & Intervisit & & Interobserver & Intervisit \\
\hline CA & 0.980 & 0.965 & & 0.992 & 0.986 \\
RA & 0.989 & 0.979 & & 0.995 & 0.993 \\
C/D ratio & 0.978 & 0.958 & & 0.989 & 0.981 \\
CV & 0.997 & 0.989 & & 0.995 & 0.994 \\
RV & 0.992 & 0.977 & & 0.994 & 0.987 \\
MCD & 0.989 & 0.995 & & 0.989 & 0.983 \\
\hline
\end{tabular}

Abbreviations: ICC, intraclass correlation coefficient; CA, cup area; RA, rim area $C / D$, cup/disk area; $C V$, cup volume; $R V$, rim volume; $M C D$, mean cup depth. evaluated here (CA, $\mathrm{RA}, \mathrm{C} / \mathrm{D}$ ratio, $\mathrm{CV}, \mathrm{RV}$, and $\mathrm{MCD}$ ), the coefficient of variation and ICC values for measurements in the sitting position made by a single observer ranged from $6.17 \%$ to $14.20 \%$ and from 0.889 to 0.964 , respectively. ${ }^{9}$ Another study showed that the interobserver and intervisit ICC values for the same six parameters measured in the sitting position ranged from 0.90 to 0.97 and from 0.86 to 0.97 , respectively. ${ }^{6}$ Our results obtained with the subjects in the sitting position are similar to those of the previous reports, further indicating that our data for the supine position can be considered as reliable as that for the sitting position.

Objective evaluation of the optic disk is especially important for infants with developmental glaucoma because of the difficulties associated with measurement of intraocular pressure and the visual field at very young ages. Moreover, cupping of the optic disk proceeds more rapidly in infants than in adults and is more likely to be reversible if the pressure is lowered early enough. ${ }^{10}$ Although we have not addressed how to obtain adequate images of the patient's eye under sedation, the methods described here may, nevertheless, be useful for monitoring these infant glaucoma patients.

This study has some limitations. The first limitation is the small number of participants; moreover, the participants in this study were younger than typical glaucoma patients. Studies including larger numbers of older participants (including glaucoma patients) will be needed to further assess the reliability of this method. Larger numbers of participants would also enable us to determine whether there is any correlation between age and CV or ICC. The second limitation (noted earlier) is that, without a way to measure optic disk parameters in sedated subjects, we were unable to obtain data from infants. For this, we may need some additional instrument(s), such as microforceps, to keep the patient's eye suitably positioned under sedation. The third limitation is that only two observers obtained disk images in this study. Studies using at least three different observers will be needed to confirm that the methods have high reproducibility. Such studies should also investigate the influence of reproducibility of the manual delineation of disk margin by the multiple observers.

\section{Conclusion}

We have established a method for optic disk evaluation by HRT II with the subjects in the supine position. The reproducibility of our measurements obtained from healthy adult subjects in the supine position was highly similar to that of measurements obtained in the sitting position. We therefore propose that our approach could prove beneficial for optic 
disk evaluation in individuals who are not able to maintain the sitting position. Modifications to this system for keeping the eye suitably positioned for optic disk evaluation in the sedated patients remain to be developed.

\section{Disclosure}

The authors report no conflicts of interest in this work.

\section{References}

1. Pederson JE, Anderson DR. The mode of progressive disc cupping in ocular hypertension and glaucoma. Arch Ophthalmol. 1980;98(3): 490-495.

2. Sommer A, Pollack I, Maumenee AE. Optic disc parameters and onset of glaucomatous field loss. II. Static screening criteria. Arch Ophthalmol. 1979;97(8):1449-1454

3. Bathija R, Zangwill L, Berry CC, Sample PA, Weinreb RN. Detection of early glaucomatous structural damage with confocal scanning laser tomography. J Glaucoma. 1998;7(2):121-127.

4. Uchida H, Tomita G, Kitazawa Y. Clinical evaluation of the Heidelberg Retina Tomograph II. Nippon Ganka Gakkai Zasshi. 2000;104(11): 826-829.
5. Harasymowycz PJ, Papamatheakis DG, Fansi AK, et al. Validity of screening for glaucomatous optic disc nerve damage using confocal scanning laser ophthalmoscopy (Heidelberg Retina Tomograph II) in high-risk populations: a pilot study. Ophthalmology. 2005;112(12): 2164-2171.

6. Altman DG, Bland JM. Measurement in medicine: the analysis of method comparison studies. Statistician. 1983;32:307-317.

7. Nakakura S, Mori E, Yamamoto M, Tsushima Y, Tabuchi H, Kiuchi Y. Intraocular pressure of supine patients using four portable tonometers. Optom Vis Sci. 2013;90(7):700-706.

8. Strouthidis NG, White ET, Owen VM, et al. Factors affecting the test-retest variability of Heidelberg retina tomograph and Heidelberg retina tomograph II measurement. Br J Ophthalmol. 2005;89(11): $1427-1432$.

9. Sihota R, Gulati V, Agarwal HC, et al. Variables affecting test-retest variability of Heidelberg Tomograph II stereometric parameters. J Glaucoma. 2002;11(4):321-328.

10. Jain MR, Marmion VJ. Rapid pneumatic and Mackay-Marg applanation tonometry to evaluate the postural effect on intraocular pressure. $\mathrm{Br} \mathrm{J}$ Ophthalmol. 1976;60(10):687-693.
Clinical Ophthalmology

\section{Publish your work in this journal}

Clinical Ophthalmology is an international, peer-reviewed journal covering all subspecialties within ophthalmology. Key topics include: Optometry; Visual science; Pharmacology and drug therapy in eye diseases; Basic Sciences; Primary and Secondary eye care; Patient Safety and Quality of Care Improvements. This journal is indexed on

\section{Dovepress}

PubMed Central and CAS, and is the official journal of The Society of Clinical Ophthalmology (SCO). The manuscript management system is completely online and includes a very quick and fair peer-review system, which is all easy to use. Visit http://www.dovepress.com/ testimonials.php to read real quotes from published authors. 